\title{
Stage IV Nasopharyngeal Keratinizing Squamous Cell Carcinoma AJCC v7
}

National Cancer Institute

\section{Source}

National Cancer Institute. Stage IV Nasopharyngeal Keratinizing Squamous Cell

Carcinoma A/CC V7. NCI Thesaurus. Code C8228.

Stage IV includes: IVA: (T4, N0, M0); (T4, N1, M0); (T4, N2, M0); IVB (Any T, N3, M0); IVC (Any T, Any N, M1). T 4: Nasopharyngeal cancer with intracranial extension and/or involvement of cranial nerves, hypopharynx, orbit, or with extension to the infratemporal fossa/masticator space. N0: No regional lymph node metastasis. N1: Nasopharyngeal cancer with unilateral metastasis in cervical lymph node(s), $6 \mathrm{~cm}$ or less in greatest dimension, above the supraclavicular fossa, and/or unilateral or bilateral, retropharyngeal lymph nodes, $6 \mathrm{~cm}$ or less in greatest dimension. Midline nodes are considered ipsilateral nodes. N2: Nasopharyngeal cancer with bilateral metastasis in cervical lymph node(s), 6 $\mathrm{cm}$ or less in greatest dimension, above the supraclavicular fossa. Midline nodes are considered ipsilateral nodes. N3: Nasopharyngeal cancer with metastasis in a lymph node (s) more than $6 \mathrm{~cm}$ in greatest dimension and/or to supraclavicular fossa. Supraclavicular zone or fossa is relevant to the staging of nasopharyngeal carcinoma and is the triangular region originally described by Ho. It is defined by three points: (1) the superior marg in of the sternal end of the clavicle, (2) the superior margin of the lateral end of the clavicle, (3) the point where the neck meets the shoulder. This would include caudal portions of levels IV and VB. All cases with lymph nodes (whole or part) in the fossa are considered N3b. M0: No distant metastasis. M1: Distant metastasis. (AJCC 7th ed.) 